\title{
Fostamatinib in chronic immune thrombocytopenia: a profile of its use in the USA
}

\author{
Kate McKeage ${ }^{1} \cdot$ Katherine A. Lyseng-Williamson $^{1}$
}

Published online: 29 August 2018

(c) Springer Nature 2018, corrected publication November/2018

\begin{abstract}
Oral fostamatinib is an orally administered small molecule spleen tyrosine kinase (SYK) inhibitor approved for the treatment of adults with chronic immune thrombocytopenia (ITP) who have an inadequate response to a previous treatment. Fostamatinib has a unique mechanism of action, whereby its active metabolite targets the SYK-mediated pathway of platelet destruction. In clinical trials, fostamatinib provided durable responses in adults with chronic ITP who had not responded or had relapsed following treatment with one or more prior ITP therapies, including corticosteroids, thrombopoietin receptor agonists, rituximab, and/or splenectomy. Most patients who respond to fostamatinib maintain platelet counts of $>50 \times 10^{9} / \mathrm{L}$ for periods of $\geq 12$ months. The most common adverse events reported with fostamatinib in clinical trials were diarrhea, hypertension, nausea, and increased transaminase levels.
\end{abstract}

Adis evaluation of fostamatinib in the treatment of

adults with chronic immune thrombocytopenia (ITP)

A small molecule spleen tyrosine kinase (SYK) inhibitor that inhibits platelet destruction via a distinct mode of action

Achieves durable responses in patients with inadequate responses to previous treatments for chronic ITP

Common adverse events include diarrhea, hypertension, nausea and increased transaminase levels

Patients should be monitored regularly throughout treatment for hypertension, hepatotoxicity, and neutropenia

\section{What is the rationale for developing fostamatinib to treat chronic ITP?}

Immune thrombocytopenia (ITP) is a heterogeneous autoimmune disorder with a variable clinical course and treatment response [1]. The pathogenesis is not fully clarified, but both antibody-mediated and/or T cell-mediated platelet destruction are involved [2, 3]. Primary ITP is defined as

Katherine A. Lyseng-Williamson

dtp@adis.com

1 Springer, Private Bag 65901, Mairangi Bay, Auckland 0754, New Zealand thrombocytopenia (platelet count $<100 \times 10^{9} / \mathrm{L}$ ) without any known cause (accounts for $\approx 80 \%$ of ITP cases), whereas secondary ITP can be attributed to a coexisting condition (e.g. systemic lupus erythematosus, antiphospholipid syndrome, Evan's syndrome, chronic lymphocytic leukemia, autoimmune lymphoproliferative syndrome following bone-marrow or solid organ transplantation, hepatitis C, HIV infection, Helicobacter pylori infection, other miscellaneous systemic infections, common variable immune deficiency, post-transfusion purpura, myelodysplasia and other conditions, as well as being induced by drugs that lead to the production of autoantibodies or occurring post-vaccination) $[3,4]$. When the disease has been present for $>1$ year, it is termed chronic ITP [4]. The most common clinical manifestations of ITP are bleeding, purpura, and fatigue. Adult patients have an increased risk of morbidity and mortality, particularly when therapy does not control platelet counts. The aim of therapy is to maintain a hemostatic platelet count (generally considered to be at least $20-30 \times 10^{9} / \mathrm{L}$ ) and minimize treatment toxicity [4].

Estimates of the incidence and prevalence of ITP in adults vary $[4,5]$. Studies indicate an annual incidence of about 1.6-3.3 per 100,000 adults and a prevalence of 4.0-23.6 per 100,000 patient-years $[4,5]$.

Because of the multifactorial pathogenesis of ITP, current treatment options are diverse [1,4]. First-line treatments are aimed at suppressing auto-antibody production and/or impeding platelet destruction, and include corticosteroids, intravenous immunoglobulin, and anti-D immunoglobulin [1, 
4]. However, first-line treatments do not often lead to durable remissions, with subsequent treatments frequently being required. Although treating corticosteroid-refractory ITP with a thrombopoietin receptor agonist (TRA; e.g. romiplostim or eltrombopag) has shown promising results [1, 4], a substantial proportion of ITP patients remain refractory to treatment. As a result, additional therapeutic approaches are needed.

One such novel approach is fostamatinib (Tavalisse ${ }^{\mathrm{TM}}$ ), which is an orally bioavailable, small molecule spleen tyrosine kinase (SYK) inhibitor that is rapidly converted to the major active metabolite R406 in the gut [6, 7]. R406 potently inhibits signal transduction of $\mathrm{Fc}$-activating receptors and $\mathrm{B}$ cell receptors, leading to reduced antibody-mediated destruction of platelets [6].

\section{For whom is fostamatinib indicated?}

In the USA, fostamantinib is approved to treat thrombocytopenia in adults with chronic ITP who have had an insufficient response to a previous treatment [8] (Table 1). Dosage adjustments are not needed in patients with renal or hepatic impairment, or those who are elderly (Table 1) [8]. According to population analyses, the pharmacokinetics of recommended dosages of fostamatinib are not altered to a clinically relevant extent by age, sex or race/ethnicity, nor are they altered to a clinically relevant extent in patients with renal or hepatic impairment $[8,9]$ (Table 1).

\section{What potential drug interactions are associated with fostamatinib?}

R406 is metabolized primarily via cytochrome P450 (CYP) 3A4 oxidization and UDP glucuronosyltransferase (UGT) 1A9 glucuronidation. Therefore, concomitant use of fostamatinib with strong CYP3A4 inhibitors may increase exposure to R406 (monitoring for fostamatinib-related toxicities is recommended) and concomitant use with strong CYP3A4 inducers may decrease exposure to R406 (concomitant use is not recommended) [Table 1] [8].

When fostamatinib is co-administered with CYP3A4, $\mathrm{BCRP}$, or P-glycoprotein substrates, exposure to the agent acting as a substrate may increase, and patients should be monitored for substrate-related toxicities (Table 1).

\section{What is the efficacy of fostamatinib in chronic ITP?}

\section{Phase 3 trials}

The efficacy of oral fostamatinib in the treatment of adults with chronic ITP was demonstrated in two randomized, double-blind, phase 3 trials (pooled results are reported) [10]. Patients were considered refractory to ITP treatment having received a median of three unique prior therapies (range 1-13), including corticosteroids, splenectomy, TRAs, and/or rituximab. At study entry, the median duration of disease was 8.7 years (range $0.3-53$ ) in fostamatinib recipients and 7.8 years (range $0.4-45$ ) in placebo recipients, and mean platelet counts in the respective groups were $16.1 \times 10^{9} / \mathrm{L}$ (range 1-51) and $19.8 \times 10^{9} / \mathrm{L}$ (range 1-156) [10]. Patients were randomized to fostamatinib $100 \mathrm{mg}$ twice daily or placebo for 24 weeks, with an option to increase the fostamatinib dosage from $100 \mathrm{mg}$ twice daily to $150 \mathrm{mg}$ twice daily after 4 weeks depending on platelet counts $(88 \%$ of patients increased their fostamatinib dosage at or after week 4) [10].

\section{Response rates and platelet counts}

A stable response by week 24 (primary endpoint; see Table 2 for definition) was achieved in significantly more patients receiving fostamatinib than receiving placebo [10]. Similarly, the proportion of patients achieving an overall response was significantly higher with fostamatinib than placebo, according to a post hoc analysis (Table 2). Among patients achieving a stable response, 15 of $18(83 \%)$ demonstrated a response at 5 of 6 clinic visits, and 14 of $18(77 \%)$ at all 6 clinic visits between weeks 14 and 24 . The median time to achieve a platelet count of $\geq 50 \times 10^{9} / \mathrm{L}$ among stable and overall responders was 15.5 and 15 days, respectively [10].

Over the 24 weeks of the trial, median platelet counts in stable and overall responders to fostamatinib were 95 and $52 \times 10^{9} / \mathrm{L}$, respectively [10]. In both the stable and overall fostamatinib responders (evaluable $n=15-18$ and $20-41$ at each time point, respectively), median platelet counts increased to $>50 \times 10^{9} / \mathrm{L}$ by week 2 , and remained $>50 \times 10^{9} / \mathrm{L}$ for all subsequent 2 -week timepoints up to week 24, with the exception of week 4 , where there was a slight decrease. In contrast, in the placebo group, median platelet counts were $<50 \times 10^{9} / \mathrm{L}$ from week 2 to 12 ( $n=32-43$ at each timepoint) [10], after which most placebo non-responders enrolled in the extension study [11].

Among patients with severe thrombocytopenia at baseline (i.e. platelet counts $<15 \times 10^{9} / \mathrm{L}$ ), an increase in platelet counts of $\geq 20 \times 10^{9} / \mathrm{L}$ to $\geq 30 \times 10^{9} / \mathrm{L}$ at weeks 12 and 24 was achieved by 21 and $15 \%$ of 47 fostamatinib recipients, respectively, compared with 5 and $0 \%$ of 21 placebo recipients [10].

A response to fostamatinib therapy occurred in all subgroups regardless of age, sex, prior therapy (splenectomy, rituximab, or TRA), baseline platelet count $(\leq 15 \times$ vs $>15 \times 10^{9} / \mathrm{L}$ ), or duration of ITP at study entry [10]. A numerically higher response to fostamatinib was achieved in younger patients (aged $<65$ years) than in older patients, and in those with platelet counts of $15-30 \times 10^{9} / \mathrm{L}$ than in those with counts of $<15 \times 10^{9} / \mathrm{L}$. 
Table 1 Summary of the US prescribing information of fostamatinib (Tavalisse ${ }^{\mathrm{TM}}$ ) in the treatment of thrombocytopenia in adults with chronic immune thrombocytopenia who have had an insufficient response to a previous treatment [8]

\section{How is fostamatinib available? \\ 100 and $150 \mathrm{mg}$ film-coated tablets to be taken with or without food}

\section{How should fostamatinib be administered?}

Initial dosage

Dosage adjustment

Maintenance dosage

Missed dose

Discontinuation after 12 weeks

Management of toxicity

\section{How should platelet counts be monitored? \\ Prior to treatment \\ During treatment}

$100 \mathrm{mg}$ twice daily for 4 weeks

$\uparrow$ to $150 \mathrm{mg}$ twice daily after 4 weeks if platelet count has not $\uparrow$ to $\geq 50 \times 10^{9} / \mathrm{L}$

Use the lowest dosage to achieve and maintain a platelet count of $\geq 50 \times 10^{9} / \mathrm{L}$

Take the next dose at its scheduled time

Discontinue if platelet count does not $\uparrow$ to a level sufficient to avoid clinically important bleeding

Modify dosage (including $\downarrow$, interruption or discontinuation) based on tolerability

$\downarrow$ from $150 \mathrm{mg}$ twice daily to $100 \mathrm{mg}$ twice daily, then to $150 \mathrm{mg}$ in the morning, then to $100 \mathrm{mg}$ in the morning as needed; if further $\downarrow$ to $<100 \mathrm{mg} /$ day are required, discontinue fostamatinib

Obtain baseline assessments

Perform monthly platelet count until a stable count of $\geq 50 \times 10^{9} / \mathrm{L}$ is achieved

Continue regular monitoring

How should fostamatinib be used in special populations?

Patients with renal or hepatic impairment, or who No specific dosage recommendations (no alterations in fostamatinib pharmacokinetics) are elderly

Pregnant women

Should not be used due to potential for fetal harm (animal data)

Breastfeeding women

Women of childbearing potential

Breastfeeding is not advised during, and for $\geq 1$ month after, treatment (potential for serious adverse reactions in the infant)

Advise the use of effective contraception during, and for $\geq 1$ month after, treatment

What are the pharmacokinetic properties of R406 (the major metabolite of fostamatinib)?

$\begin{array}{ll}\text { Absolute bioavailablity } & 55 \% \\ \text { Median time to } \mathrm{C}_{\max } & \approx 1.5 \mathrm{~h} \text { (range } 1-4 \mathrm{~h}) \\ \text { Plasma protein binding } & 98.3 \% \text { in vitro } \\ \text { Mean volume of distribution } & 256 \mathrm{~L} \text { at steady state } \\ \text { Terminal elimination half-life } & \approx 15 \mathrm{~h} \\ \text { Excretion } & 80 \% \text { in the feces; } \approx 20 \% \text { in the urine }\end{array}$

What clinically relevant drug interactions may potentially occur with fostamatinib?

Strong CYP3A4 inhibitor (e.g. ketoconazole)

Monitor for fostamatinib-related toxicities (exposure to R406 may $\uparrow$ )

Concomitant use not recommended (exposure to R406 may $\downarrow$ )

Monitor for toxicities of the substrate (concentrations of the substrate may $\uparrow$ )

Substrates of CYP3A4 (e.g. simvastatin), BCRP

(e.g. rosuvastatin) and P-gp (e.g. digoxin)

$C_{\text {max }}$ maximum plasma concentration, $C Y P$ cytochrome P450, $P$-gp P-glycoprotein, $\uparrow$ increase(d), $\downarrow$ decrease/reduction

\section{Bleeding-related events and rescue medication use}

None of the patients with an overall response to fostamatinib experienced a bleeding-related serious adverse event compared with $10 \%$ of placebo recipients, and $7 \%$ of patients without an overall response to fostamatinib (Table 2) [10]. Relative to both the placebo group and the subgroup without an overall response to fostamantinib, numerically fewer fostamatinib recipients with an overall response experienced a moderate or severe bleeding-related adverse event or received rescue therapy (e.g. corticosteroids, platelet transfusion) [Table 2] [10].

\section{Long-term extension study}

One hundred and twenty-three patients in the phase 3 trials received fostamatinib in an open-label, long-term extension [11]. Most patients who did not respond to fostamatinib, and most placebo recipients, entered the extension at week 12 of the phase 3 trials. A stable response to fostamatinib was defined as a platelet count $\geq 50 \times 10^{9} / \mathrm{L}$ at 4 of 6 evaluations during weeks $14-24$ of the phase 3 trials, or $\geq 50 \times 10^{9} / \mathrm{L}$ within 12 weeks of starting therapy without the need for rescue therapy and $\geq 50 \times 10^{9} / \mathrm{L}$ at two of three subsequent monthly evaluations during the extension study [11]. 
Table 2 Efficacy of oral fostamatinib in patients with chronic immune thrombocytopenia in the pooled analysis of two randomized, doubleblind, 24-week phase 3 trials [10]

\begin{tabular}{|c|c|c|c|c|}
\hline \multirow[t]{2}{*}{ Outcome } & \multicolumn{2}{|c|}{ Total study population } & \multicolumn{2}{|c|}{ FOS subgroups based on overall response to FOS } \\
\hline & $\begin{array}{l}\text { FOS } \\
(n=101)\end{array}$ & $\begin{array}{l}\text { PL } \\
(n=49)\end{array}$ & $\begin{array}{l}\text { With an overall response } \\
(n=43)\end{array}$ & $\begin{array}{l}\text { Without an overall response } \\
(n=58)\end{array}$ \\
\hline Stable response $\mathrm{e}^{\mathrm{a}}(\%$ of pts) & $18^{*}$ & 2 & & \\
\hline Overall response ${ }^{\mathrm{b}}$ (\% of pts) & $43^{*}$ & 14 & & \\
\hline Bleeding-related serious $\mathrm{AE}$ (\% of pts) & & 10 & 0 & 7 \\
\hline Bleeding-related moderate-to-severe $\mathrm{AE}$ (\% of pts) & & 16 & 9 & 10 \\
\hline Use of rescue medication (\% of pts) & & 45 & 16 & 34 \\
\hline
\end{tabular}

$A E$ adverse event, FOS fostamatinib, PL placebo, pts patients

$* p<0.01$ vs PL

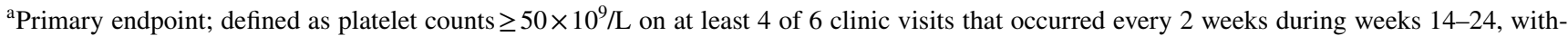
out requiring rescue therapy after week 10

${ }^{\mathrm{b}}$ Defined as $\geq 1$ platelet count $\geq 50 \times 10^{9} / \mathrm{L}$ during weeks $0-12$; determined in a post hoc analysis

Most patients who achieved a stable response to fostamatinib were able to maintain a stable response for $\geq 12$ months, and up to $>24$ months in some individuals [11]. Among 117 patients who received fostamatinib for $\geq 12$ months, 17 of $25(68 \%)$ maintained a stable response, with a median platelet count of 111 and $115 \times 10^{9} / \mathrm{L}$ after 12 and 52 weeks of treatment, respectively (median platelet count prior to fostamatinib was $23 \times 10^{9} / \mathrm{L}$ ) [11]. Although the Kaplan-Meier median duration of a stable response for the 17 patients who maintained a response has not yet been reached, it is estimated to be $>28$ months (range 12 to $>28$ months) [11]. Bleeding-related serious adverse events occurred in $9 \%$ of patients, with all but one of these events occurring in patients without a stable response.
Among patients who received placebo during the phase 3 studies and then switched to fostamatinib, 10 of 44 (23\%) achieved a stable response compared with one (2\%) patient during the placebo phase $(p<0.01)[11]$.

\section{What is the tolerability profile of fostamatinib?}

In the 24-week phase 3 trials in adults with chronic ITP, treatment-emergent adverse events (TEAEs) were reported in 83 and $75 \%$ of patients in the fostamatinib (100 or $150 \mathrm{mg}$ twice daily) and placebo groups, respectively (pooled data), with the majority of these being mild (39 and 56\% of TEAEs in the

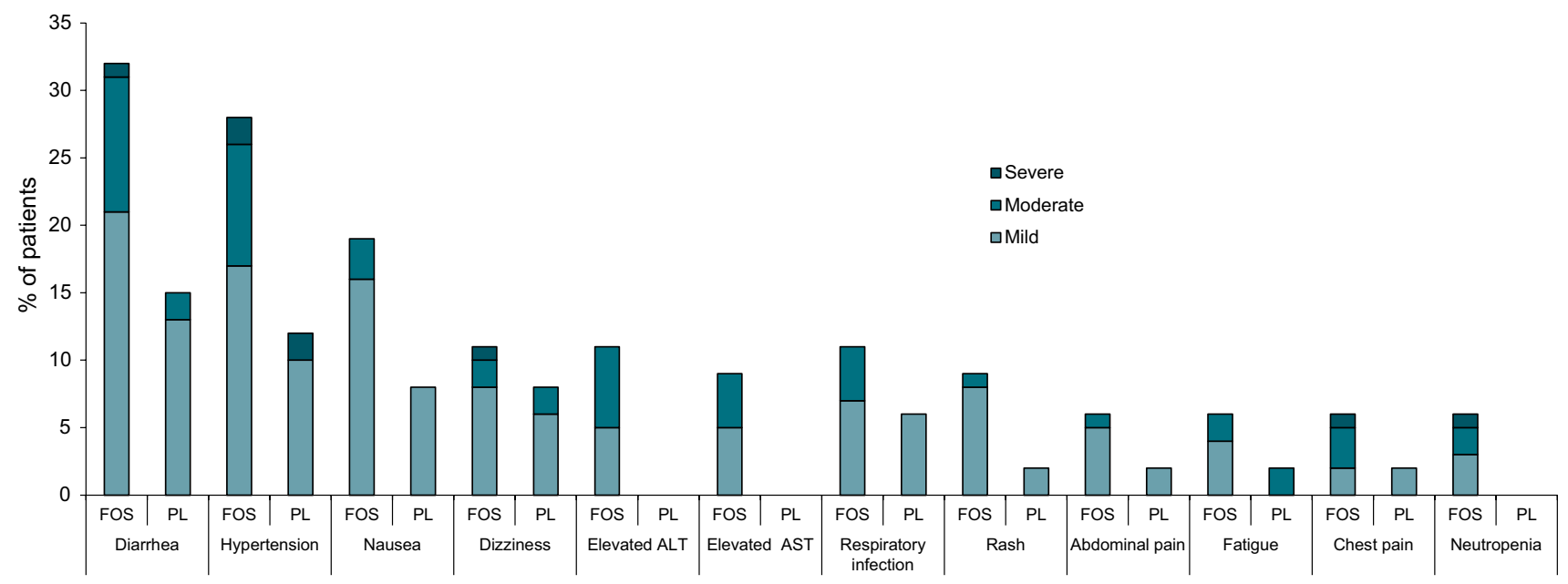

Fig. 1 Treatment-emergent adverse events reported in $>5 \%$ of 102 fostamatinib (FOS) recipients and at a numerically higher incidence than in 48 placebo (PL) recipients in two pooled phase 3 trials in patients with chronic immune thrombocytopenia [10]. Diarrhea includes diarrhea and frequent bowel movement; hypertension includes hypertension, blood pressure (BP) increased, diastolic BP
(DPB) abnormal, and DBP increased; respiratory infection includes respiratory tract infection (RTI), upper RTI, lower RTI, and viral upper RTI; rash includes rash, rash erythematous and rash macular; abdominal pain includes abdominal pain, and abdominal pain upper; neutropenia includes neutropenia and neutrophil count decreased 


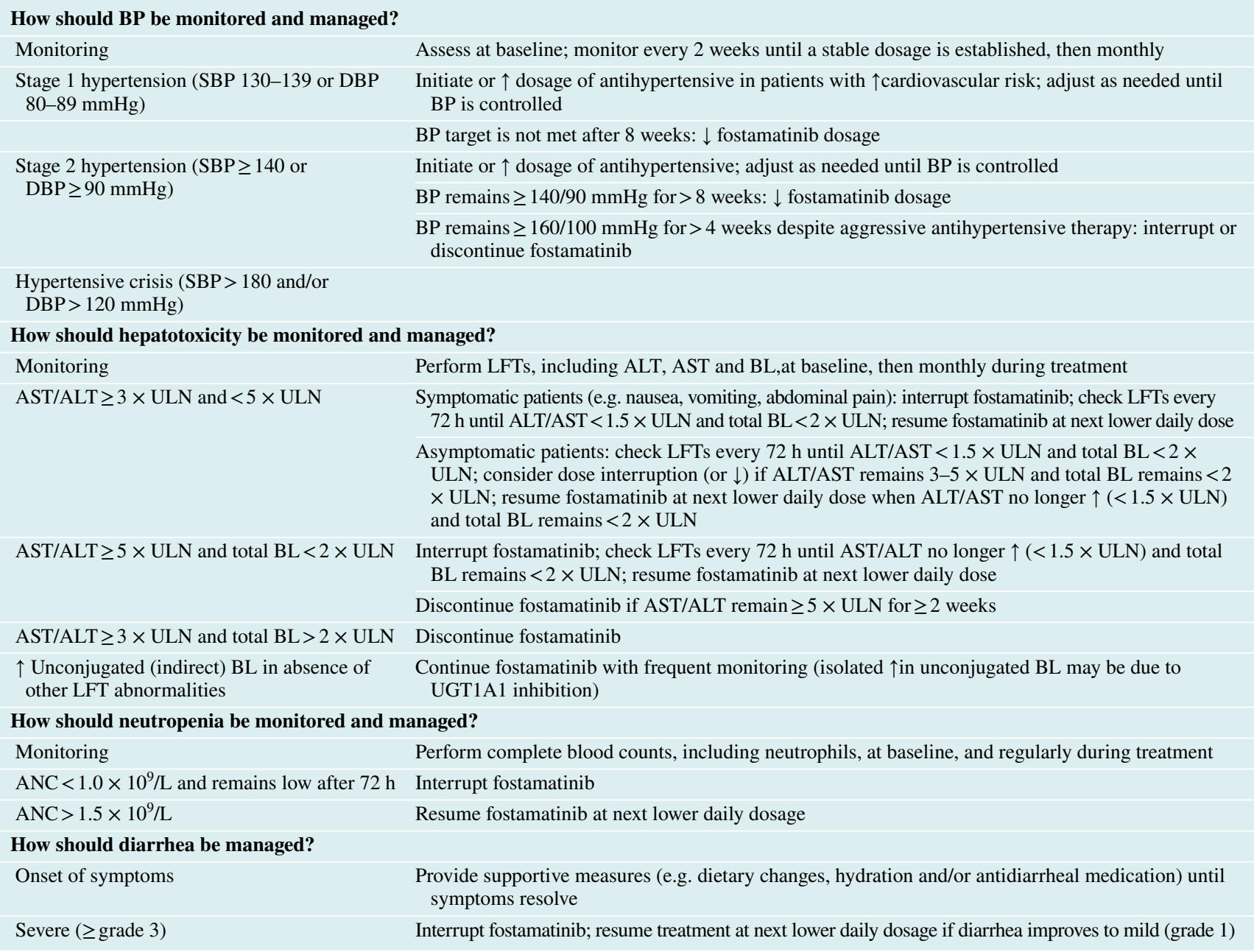

$A N C$ absolute neutrophil count, $B P$ blood pressure, $B L$ bilirubin, $D B P$ diastolic BP, $L F T$ liver function test, $S B P$ systolic BP, $U G T$ glucuronosyltransferase, $U L N$ upper limit of normal, $\uparrow$ increase(d)/elevated, $\downarrow$ decrease

fostamatinib and placebo groups, respectively) or moderate (42 vs $25 \%$ ) in severity [10]. Figure 1 shows the most commonly reported categories of TEAEs and their severity [10].

In the pooled fostamatinib and placebo groups, TEAEs led to discontinuation in generally similar proportions of patients (10 vs $8 \%$ ), although the proportions requiring dose reductions ( 9 vs $2 \%$ ) or interruptions (18 vs $10 \%$ ) resulting from an adverse event were numerically higher in the fostamatinib group [10]. The most common TEAEs (incidence of $\geq 2 \%$ ) that led to fostamatinib dose reductions or interruptions were hypertension, diarrhea, increased ALT levels, and influenzalike illness. Three severe TEAEs led to treatment withdrawal in fostamatinib recipients, including one case each of nonserious chest pain and syncope, pneumonia, and thrombocytopenia. No patients who achieved a stable response, and three patients who achieved an overall response, discontinued fostamatinib therapy due to a TEAE [10].

Serious TEAEs were reported in 13 and $21 \%$ of patients in the fostamatinib and placebo groups, respectively; however, the serious events were consider to be related to treatment in only 4 and $2 \%$ of patients in the respective groups [10].

In the long-term extension study, $75 \%$ of 123 patients experienced at least one TEAE, and $52 \%$ of patients had at least one treatment-related adverse event [11]. The most frequent TEAEs were diarrhea ( $28 \%$ of patients), hypertension $(15 \%)$, petechia (15\%) and epistaxis (14\%). Most TEAEs were mild to moderate in severity, with $22 \%$ of patients experiencing a serious TEAE event. A TEAE led to treatment withdrawal in 15 (12\%) patients, including diarrhea in five patients, liver enzyme elevations in three, and neutropenia in two (all other events were reported in a single patient).

\section{Adverse effects of special interest}

Fostamatinib is associated with increases in blood pressure (BP; Fig. 1) [8]; therefore, BP should be regularly monitored in all patients, and hypertension managed based on the PI recommendations (Table 3) [8]. Increases in BP are 
thought to result from the inhibitory effect of fostamatinib on vascular endothelial growth factor receptor signaling leading to reduced nitric oxide release from the endothelium [12]. After administration of fostamatinib $100 \mathrm{mg}$ twice daily for 28 days, mean placebo-adjusted increases in systolic and diastolic BP were 2.93 and $3.53 \mathrm{mmHg}$, respectively [8]. Blood pressures $\geq 140 / 90 \mathrm{mmHg}$ were recorded in 31 and $15 \%$ of patients in the fostamatinib and placebo groups, respectively. Among 19 fostamatinib recipients with $\mathrm{BP} \geq 140 / 90 \mathrm{mmHg}, \mathrm{BP}$ returned to baseline levels within one week of discontinuing fostamatinib in 11 (58\%) patients.

Fostamatinib is also associated with an increased risk of hepatotoxicity (Fig. 1). Liver function tests should be performed regularly, and PI recommendations for the management of hepatotoxicity followed (Table 3). As R406 acts as an inhibitor of UGT1A1, increases in unconjugated bilirubin may occur in the absence of other liver function test abnormalities [8].

Neutropenia and diarrhea have also been associated with fostamatinib (Fig. 1). Complete blood counts, including neutrophil counts, should be performed regularly, and PI recommendations for the management of neutropenia and diarrhea should be followed (Table 3) [8].

\section{What conclusions can be made regarding the use of fostamatinib in chronic ITP?}

Oral fostamatinib is an option for the treatment of chronic ITP in adults who have had an insufficient response to a previous treatment. In clinical trials, fostamatinib provided durable responses in adults with chronic ITP who had not responded or relapsed following treatment with one or more prior ITP therapies, including corticosteroids, TRAs, rituximab, and/or splenectomy $[10,11]$. Unlike other ITP therapies, fostamatinib has a unique mechanism of action, targeting the SYK-mediated pathway of platelet destruction [6]. Most patients who respond to fostamatinib maintain platelet counts of $>50 \times 10^{9} / \mathrm{L}$ for periods of $\geq 12$ months [11]. The most common adverse events reported with fostamatinib in clinical trials were diarrhea, hypertension, nausea, and increased transaminase levels [10]; monitoring is required throughout treatment because of the risk of hypertension, hepatotoxicity, and neutropenia (Table 3) [8]. Further clinical trial data should help establish if administering fostamatinib earlier in the course of the disease could improve response rates, as well as the comparative efficacy of fostamatinib versus other ITP therapies [10]. Identifying the patient populations who are most likely to respond to fostamatinib may also potentially improve treatment outcomes [10].

Acknowledgements The manuscript was reviewed by: W. Homenda, Wojewódzki Szpital Specjalistyczny im. J. Korczaka i Akademia Pomorska w Słupsku, Slupsk, Poland; A. A. Khalafallah, Menzies
Institute for Medical Research and Faculty of Health Sciences, University of Tasmania, Launceston, TAS, Australia. During the peer review process, Rigel Pharmaceuticals Inc., the marketing-authorization holder of fostamatinib, was also offered an opportunity to provide a scientific accuracy review of their data. Changes resulting from comments received were made on the basis of scientific and editorial merit.

\section{Compliance with ethical standards}

Funding The preparation of this review was not supported by any external funding.

Conflict of interest K. McKeage and K.A. Lyseng-Williamson are employees of Adis/Springer, are responsible for the article content and declare no conflicts of interest.

Open Access This article is distributed under the terms of the Creative Commons Attribution-NonCommercial 4.0 International License (http://creativecommons.org/licenses/by-nc/4.0/), which permits any noncommercial use, duplication, adaptation, distribution and reproduction in any medium or format, as long as you give appropriate credit to the original author(s) and the source, provide a link to the Creative Commons license and indicate if changes were made.

\section{References}

1. Niscola P, Scaramucci L, Giovannini M. Spleen tyrosine kinase inhibition: a new promising approach to chronic and refractory immune thrombocytopenia. Immunotherapy. 2018;10(1):5-7.

2. Zufferey A, Kapur R, Semple JW. Pathogenesis and therapeutic mechanisms in immune thrombocytopenia (ITP). J Clin Med. 2017;6(2):16.

3. Cines DB, Bussel JB, Liebman HA, et al. The ITP syndrome: pathogenic and clinical diversity. Blood. 2009;113(26):6511-21.

4. Kistangari G, McCrae KR. Immune thrombocytopenia. Hematol Oncol Clin N Am. 2013;27(3):495-520.

5. Terrell DR, Beebe LA, Vesely SK, et al. The incidence of immune thrombocytopenic purpura in children and adults: a critical review of published reports. Am J Hematol. 2010;85(3):174-80.

6. Braselmann S, Taylor V, Zhao H, et al. R406, an orally available spleen tyrosine kinase inhibitor blocks fc receptor signaling and reduces immune complex-mediated inflammation. J Pharmacol Exp Ther. 2006;319(3):998-1008.

7. Baluom M, Grossbard EB, Mant T, et al. Pharmacokinetics of fostamatinib, a spleen tyrosine kinase (SYK) inhibitor, in healthy human subjects following single and multiple oral dosing in three phase I studies. Br J Clin Pharmacol. 2013;76(1):78-88.

8. Tavalisse ${ }^{\mathrm{TM}}$ (fostamatinib disodium hexahydrate): US prescribing information. South San Francisco: Rigel Pharmaceuticals Inc. 2018.

9. Martin P, Oliver S, Gillen M, et al. Pharmacokinetic properties of fostamatinib in patients with renal or hepatic impairment: results from 2 phase I clinical studies. Clin Ther. 2015;37(12):2823-36.

10. Bussel J, Arnold DM, Grossbard E, et al. Fostamatinib for the treatment of adult persistent and chronic immune thrombocytopenia: results of two phase 3 , randomized, placebo-controlled trials. Am J Hematol. 2018;93(7):921-30.

11. Bussel JB, Arnold DM, Cooper N, et al. Long-term maintenance of platelet responses in adult patients with persistent/chronic immune thrombocytopenia treated with fostamatinib: 1-year efficacy and safety results [abstract]. Blood. 2017;130(Suppl 1):16.

12. Skinner M, Philp K, Lengel D, et al. The contribution of VEGF signalling to fostamatinib-induced blood pressure elevation. $\mathrm{Br} \mathrm{J}$ Pharmacol. 2014;171(9):2308-20. 